\title{
Research and Implementation of QoS Route Based on Overlay Network
}

\author{
Lei Bai, Fuling Li \\ North China Institute of Science and Technology, Beijing 101601, China \\ huner2011@foxmail.com
}

\begin{abstract}
Keywords: Overlay network, Mobile Ad Hoc Network Control System, QoS route, QAODV, C-QAODV
\end{abstract}

\begin{abstract}
QAODV based on AODV routing protocol usually works well, but it has the problem of extendibility. When there are many network nodes, the routing control message including RREQ increases and the performance of protocol reduces. In order to reduce control message, the paper combines the idea of QAODV and cluster, and proposes an incremental clustering routing protocol based on QAODV, C-QAODV(Clustering QAODV). The protocol writes the code on NS2 simulation platform, which verifies the extendibility.
\end{abstract}

\section{Introduction}

Overlay network is established on a or several existing networks. It is a virtual communication structure and logically covers the physical network. The extra, indirect and virtual layers are increased to improve some attributes of infrastructure network and improve the performance of network. Overlay network has the advantages of easy implementation, good expansibility, great flexibility and robustness. The introduction of Overlay network may increase average delay, but it not only can change the delay and the distribution of packet loss, and increase connectivity of nodes, but also can provide service quality ( QoS ) mechanism, and improve the control system performance. The algorithm performance of the route can be analyzed by simulation tool. And Overlay technique can be used. And interdependent wireless fidelity is organized into MANET based on Overlay to implement NCS application. The paper researches from the perspective of QoS route. Introducing Overlay technique can ensure that more businesses are deployed under the situation that the existing bottom-layer physical network has no change, which is easy to apply the algorithm.

\section{Introduction of Overlay Network}

Overlay is a network which is established on the existing physical network by increasing extra, indirect and virtual layer. It is a method of constructing network, and has no relationship with specific technique and layer. It is used to improve the features and improve the performance of network.

Overlay network has the following features. Firslty, Good expansibility. An Overlay network has no need to change the existing network structure and doesn't require the support of network components in the network. And the implementation of the idea can speed up new network functions and the deployment of service. Secondly, great flexibility. Overlay network establishes an abstract connection. And the connection can be optimized according to the requirements of applications. Thirdly, robustness. Overlay network is more robust than network facilities of the bottom layer.

\section{QAODV Working Mechanism}

Routing detection. Routing detection of QAODV is established on the basis of requirements and limited flooding. When a node needs to communicate with other nodes, and there is no effective path, the routing detection will be started. The node broadcasts a route request packet RREQ including the requested bandwidth Bmin, end-to-end delay Tmax requirement, source node address, source node serial number and broadcast ID. The middle node receiving the route request packet 
only is processed when the group is received for the first time., executing bandwidth allow control process. Once the request is accepted, if the node has no route entry of destination mode, a new route entry is added to the routing list. The value of state field explored and counts is 0 . If the node has the route entry to destination node, the state field of the entry is changed into explored, and counts value has no change. And a reverse route is added to the routing list without resource control, the reason for which is that the route is only used to return RREP group. The node continues to broadcast the request to the next hot until to the destination node. The node only reserves the time of routing entrance entry 2Tmax which is marked as explored. If the response packet is not received beyond the time, the routing entry is deleted or changed into activated. The method can reduce the load of control message and ineffective information of routing list.

Route registration. In the process of routing detection, several routing request packets can reach the destination node from different paths. For the received request packets, destination nodes can send the response packets to the source node along reverse path. If the received middle node makes routing detection, and the state is explored, the present bandwidth will be examined if it meets QoS requirement (network dynamic change, route request packet may be not equal to the present bandwidth). If it accepts, the routing entry is upgraded and is marked as registration, and the node prepares to receive the real business flow. However, the reserved bandwidth is only activated when the first data packet reaches, and counts value is added 1, and the state field is changed to be activated. If there is no data packet reaching in time interval, it means that source node doesn't choose the path, and the registration route entry is ineffective.

Route maintenance. The movement of nodes makes QoS route which meets the requirements originally not meet the requirement. Therefore, there is need to maintain the established route.

(1)Route interrupt recovery. When the node discovers link interruption, it broadcasts RERR subgroup to inform all upstream nodes, and deletes all route entries or changes into ineffective state. In figure 1, when R2 detects that R3 is not in the transmission range, all route entries whose rt_nexthop is R3 in routing list of R2 is changed into ineffective, and rt_dst is added to unreachable_dst of RERR subgroup for broadcast. When R1 receives the broadcast subgroup RERR of R2, the routing list is examined if there is routing entry from R2 to unreachable_dst. If there is the route entry, it is ineffective, and resources are released to continue to broadcast. Finally, the source node S receives RERR subgroup of R1, and the links from R1 to D1 is set to be ineffective.

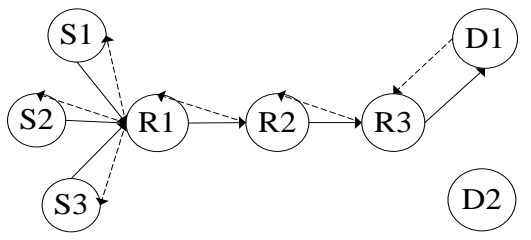

Figure 1 Route example

(2) Delay interrupt recovery. The movement of nodes makes the end-to-end delay can’t meet the requirements, which needs the corresponding process. It is complicated to maintain the delay between adjacent nodes in the network, and there is no effective method. And the judgment of end-to-end delay is put on destination node. Literature [21] proposes a method of estimating synchronous time difference of source node and destination node, and the data packet can be added to Time $\mathrm{T}$ and Toffset of source node when source node sends data packet. When destination node receives the data packet, it is easy to compute the actual end-to-end delay of the packet, and if it can meet the requirements is judged. When it doesn't meet the requirements, the links may be interrupted. So unicast is not used. And a route is broadcast to the source node to update the packet, and the packet format is the same to RREP. And the reserved fields can be used for differentiation. The processing way after middle node receives the packet is the same to RREP. When it reaches source node, the source node makes routing detection again. The release of path resource is equal to the reserved time interval of a resource, Tinterval. 


\section{Working Mechanism of C-QAODV}

According to the function and position of nodes in the network, the state of nodes can be divided into un-assigned which has no cluster, Cluster Head which is the representative of cluster member participating in data forwarding, Gate Way which belongs to many cluster heads, Cooperation Gate Way which needs bigeminy to realize triple jump of two cluster heads, and Cluster member, as shown in Fig. 2.

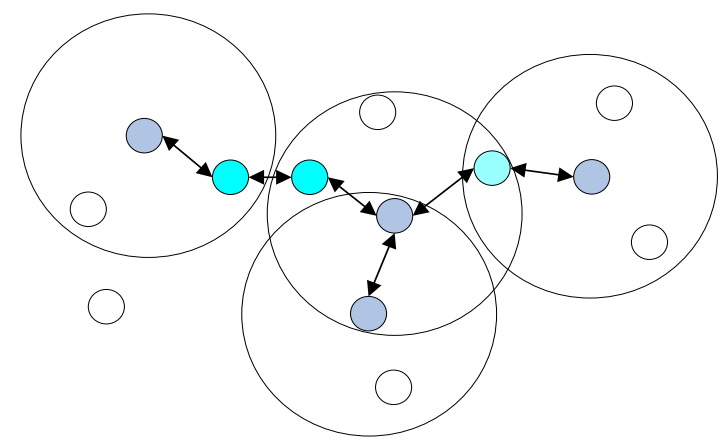

Fig. 2 Node distribution

Each node has its own IP address NodeIP, Cluster State, CHList of cluster of nodes, NbList and GWList. GWList stores all gateway information of clusters of nodes, and each entry of each list includes network gateway node IP address GWIP, GWType, CHIP1 and CHIP2. If a node is cluster head node, it includes NbCHList and ClusterNodeList. In NbCHList, each item includes NbCHIP, GWType and GWIP. If the field value of GWType is CGW, IP address of network gateway is CGWIP1 and CGWIP2. If the field value of GWType is $\mathrm{CH}$, it means that two cluster heads are adjacent. And IP address of network gateway node is the same to NbCHIP, IP address of another cluster head. In Cluster Node List, each entry includes Cluster Node IP and Type of cluster member.

\section{Research on Implementation Mechanism of Protocol}

Implementation mechanism of QAODV. Route detection and maintenance of QAODV is based on event. The implementation needs to determine when to stimulate QAODV route event, which includes when to stimulate route request, when and how to cache data packet, and when to generate RERR if there is no an effective route. For implementation, using Snooping to inquiry, modifying system kernel or Nerfilter technique can achieve the triggering time of the events.

Netfilter is some mount points in linux protocol stack. The custom codes of uses can be used to redirect packet streams. And the codes can guard process detection, discard, modification or queuing up of the data packets for user layer. The method of using Netfilter for inquiry is similar to that of Snooping, but it doesn't depend on ARP packet. Compared with others, they have many advantages, as follows. There is no unnecessary communication, there is great universality and easy installation. Therefore, the mechanism is applied in the implementation. The hitch of Netfilter is used to redirect the packet, and it accepts the packets which are sent to other machines (NF_IN_POST_ROUTING) from (NF_IP_LOCAL_OUT) and (NF_IP_PRE_ROUTING) . The hitching functions are used by QAODV kernel module. Ip_queue module is used to queue up the packets in the guarding process of user space. QAODV daemon process handles the packets. And structure model is shown in Fig. 3. 


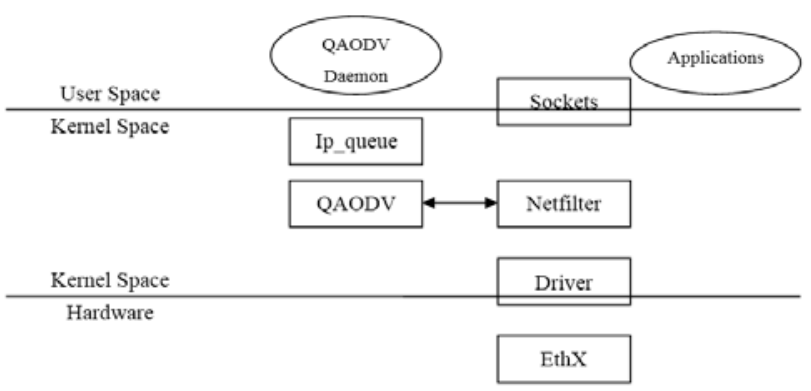

Fig. 3 Netfilter implementation structure

The implementation structure of C-QAODV is similar. The implementation mechanism of two algorithms and data structure of message is described in the above chapter. And the implementation can refer to implementation codes in NS simulation software, and the writing of codes needs to be researched.

Netfilter framework. Netfilter has the following features.

(1) Each protocol defines hooks, and IPv4 defines 5 hooks which goes through the process of packet transmission in protocol stack. On each node, the protocol applies the packets and hook number to call Netfilter framework.

(2) Some kernels can monitor different hooks for each protocol after registration. Therefore, when the packet goes throught the Netfilter framework, it examines if there is modules as protocol and hook registration. There are the modules, they have chance to examine, abandon, and allow the packets, or request Netfilter as user space queuing packet.

(3) The queued packets can be collected and sent to user space, and the packets are processed asynchronously.

(4) There are good codes and documents, which has great significance for an open framework with good expansibility. If there is no the feature, Netfilter structure is difficult to be operated.

The process of data packet going through Netfilter is shown in Fig. 4.

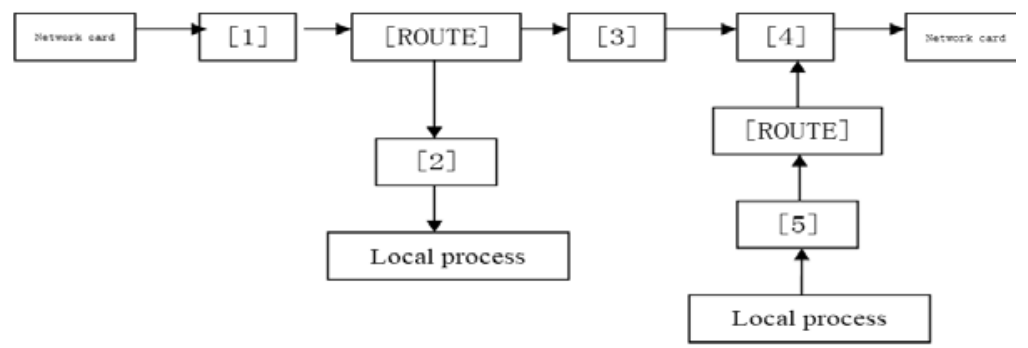

Fig. 4 Schematic diagram of data packet going through Netfilter system

[1]: NF_IP_PRE_ROUTING: the data packets which just enter network layer pass the point ( just completing the detection of version number and verification), and the source address is transited on the point.

[2]: NF_IP_LOCAL_IN : after routing lookup, the data packets which are sent to the computer pass the examination point, and INPUT packet is filtered on the point.

[3] : NF_IP_FORWARD: the packets to be forwarded pass the examination point, and FORWORD packet is filtered on the point.

[4]: NF_IP_POST_ROUTING: the packets which are to be sent out through the network facility pass the examination point, and the transformation function of the built-in destination address (including address spoofing) is implemented on the point.

[5] : NF_IP_LOCAL_OUT : the packets which are sent out by the computer pass the examination point, and OUTPUT packets are filtered on the point.

\section{Conclusions}

The paper selects QoS routing mechanism of MANET as research object. Based on QoS routing protocol, the paper proposes a new QoS routing protocol based on AODV, QAODV. The protocol 
uses the mechanisms including bandwidth access control, end-to-end delay control and resource reservation/release, which provides Qos support with bandwidth and delay as indicators for real-time business flow. The paper proposes a cluster routing protocol based on QAODV, C-QAODV. The working mechanism, establishment and maintenance of cluster is analyzed, and the simulation program is designed on NS platform and receives simulation experiment. We find that the performance of C-QAODV is similar to that of AODV when there are fewer network nodes and the load is lower. But when there are more nodes and the load is heavy, the performance of C-QAODV is evidently better than that of AODV.

\section{References}

[1] RSVP-TE: Extensions to RSVP for LSP Tunnels. IETF work in progress, February 2000

[2] Zhang W., Branicky M. S., and Philips S. M., Stability of networked control systems, IEEE Control Systems Magazine, 2001, 21(1).pp.84 99

[3] T.C. Yang. Networked control system: a brief survey. IEE Proceedings-Control Theory and Applications, 153(4): 403 - 412, July 2006.

[4] M.B.G. Cloosterman, N. van de Wouw, W.P.M.H. Heemels, H. Nijmeijer, Robust Stability of Networked Control Systems with Time-Varying Network-Induced Delays, in 45th IEEE conference on decision and control; San Diego, CA, United States, 4980-4985, 2006

[6] Bu, S \& Naghdy, F, Wireless ad-hoc control networks, 3rd IEEE International Conference on Industrial Informatics (INDIN '05), 10-12 August 2005, 839-844.

[7] Kawka P A, Alleyne A G. Stability and feedback control of wireless networked systems[C].USA Portland: American Control Conference,2005.

[8] G. Nikolakopoulos A. Panousopoulou and A. Tzes, MANET-Issues and their effects on control applications, 10th IEEE International Conference on Emerging Technologies and Factory Automation, Catania, Italy, 19-22 September, 2005.

[9] Colandairaj, J.; Scanlon, W.; Irwin, G.. Understanding wireless networked control systems through simulation. IEEE: Computing \& Control Engineering Journal. Vol.16, Issue 2, April-May 2005 pp: $26-31$ 